\title{
Impact of Battery Ageing on an Electric Vehicle Powertrain Optimisation
}

\author{
Daniel J. Auger", Maxime F. Groff, Ganesh Mohan, Stefano Longo and \\ Francis Assadian
}

Centre for Automotive Engineering, Cranfield University, Bedfordshire, United Kingdom e-mail: d.j.auger@cranfield.ac.uk

\begin{abstract}
An electric vehicle's battery is its most expensive component, and it cannot be charged and discharged indefinitely. This affects a consumer vehicle's end-user value. Ageing is tolerated as an unwanted operational side-effect; manufacturers have little control over it. Recent publications have considered trade-offs between efficiency and ageing in plug-in hybrids (PHEVs) but there is no equivalent literature for pure EVs. For PHEVs, battery ageing has been modelled by translating current demands into chemical degradation. Given such models it is possible to produce similar trade-offs for EVs. We consider the effects of varying battery size and introducing a parallel supercapacitor pack. (Supercapacitors can smooth current demands, but their weight and electronics reduce economy.) We extend existing EV optimisation techniques to include battery ageing, illustrated with vehicle case studies. We comment on the applicability to similar EV problems and identify where additional research is needed to improve on our assumptions.
\end{abstract}

\section{KEYWORDS}

Electric vehicles, Battery, Ageing, State-of-health, Power train optimisation.

\section{INTRODUCTION}

\section{Motivations}

There can be very few people in the developed world who have not heard of 'global warming' or climate change, and there is a strong scientific consensus that human activity is one of the major drivers. The UK's Royal Society has published a report [1] summarizing the current state of knowledge: this report notes that present-day $\mathrm{CO}_{2}$ concentrations are higher than at any time in the past 800,000 years that there is 'strong evidence that changes in greenhouse gas concentrations due to human activity are the dominant cause of the global warning that has taken place over the last half century'. The Royal Society is far from alone: NASA maintains a detailed web site [2] documenting the evidence for climate change and the overwhelming consensus for it. Controlling and reducing $\mathrm{CO}_{2}$ emissions is a high priority for government and society: it is reasonable to argue that it is the greatest challenge of our time.

There are many sources of $\mathrm{CO}_{2}$ emissions, but road transport accounts for a big part. In 2007, the European Parliament [3] noted that cars and light commercial vehicles were responsible for $19 \%$ of the EU's $\mathrm{CO}_{2}$ emissions and $60 \%$ of its oil consumption. In 2011 ,

\footnotetext{
* Corresponding author
} 
the UK's domestic transport (which includes road, rail and air) accounted for nearly a quarter of the country's $\mathrm{CO}_{2}$ emissions and the UK government [4] believes that in order to meet 2030 targets, 'average new car' emissions will need to fall from their present levels to $50-70 \mathrm{gCO}_{2} / \mathrm{km}$. (The targets for vans are similarly ambitious.) These reductions represent an approximate halving of present-day emissions. To solve this problem, we need clean propulsion technologies. Among the technologies being explored by vehicle manufacturers are plug-in hybrids (PHEVs) and electric vehicles (EVs).

PHEVs and EVs have made some sales, but they are yet to account for a significant share of the market. It was reported in the UK press [5] that in the first three quarters of 2012, only 1419 electric cars were sold, a 'fraction' of the UK's two-million-car market. There are many suspected factors in the reluctance of consumers to switch to the new technology-range anxiety, for example, and worry about the availability of charging points, just to name a couple. Perhaps the most significant factors are cost and perceived reliability. It is easy to see that these are very real market concerns by looking at manufacturer's prices and the existence of 'battery rental' schemes. One manufacturer [6] charges approximately $\$ 37,700$ (before subsidy) for its A-segment $\mathrm{EV}$, and just under $\$ 12,500$ for its closest petrol equivalent. Another [7] charges just under $\$ 40,600$ (before subsidy) for its cheapest $\mathrm{C}$-segment EV: by contrast, the closest petrol-driven equivalent sells for just under $\$ 18,700$; the same manufacturer also offers a battery rental scheme, under which the up-front cost of the EV is reduced by $\$ 7,800$. The battery is clearly expensive, and there is also a clear hint that some consumers fear it may not last. Similar fears are not apparent with internal combustion engines.

Manufacturers have been working with internal combustion engines for decades, and, in con-sequence, they have a very good understanding of the way components will age, wear and fail: it is relatively easy to design a traditional car, confident that it will last, say, ten years or $200,000 \mathrm{~km}$. Traditionally, when a manufacturer introduces something new, it is done through 'incremental change'. The new component accounts for a relatively small part of the vehicle's overall cost, so if a component is likely to fail, the associated cost will not be too severe. When considering a hybrid or EV, however, this is not the case: electric vehicle technology is radically different from the technologies manufacturers are used to, and consequently, it presents a big risk.

\section{Traction batteries and ageing}

As far as automotive manufacturers are concerned, high-power batteries are the biggest un-known, and the chance of premature failure is seen as being high. Since the batteries are the most expensive part of the car-comparable to a traditional vehicle's engine in importance-the risk associated with the batteries is considered extremely high. Manufacturers have to make an unpleasant choice between (a) passing the risk directly to the purchaser, or (b) increasing their prices to cover the cost of the providing generous warranty cover. The more manufacturers can understand about what might age a battery, the better, particularly if this leads to new ways to extend the operational lifetime. Although there is undoubtedly an important place for battery chemistry in this, we need to make sure this is in the right context: we need to know how the battery is affected by the demands placed on it in a real-world driving scenario. Once we know this, we can predict how long a given design will last in service.

In 2011, a consortium of British universities began work on the four-year 'FUTURE Vehicles' project [8], which aims to develop the fundamental understanding of components that are key to hybrid and electric vehicles. The project contains several work packages which are running in parallel. One work package aims to develop a new deep understanding of the fundamentals of battery chemistry. This work package has pub- 
lished results [9] and models are rapidly developing to the point of usefulness. Our own work package aims at understanding how we will be able to use these new models to understand battery ageing in the context of the vehicle, and how we can apply control and optimisation techniques to mitigate the effects of this ageing. While we wait for our colleagues' models to mature, we have begun to work using models derived from the existing literature.

To date, some work has been done by US researchers [10] understanding the relationship be-tween usage and ageing, though it has focussed on plug-in hybrids (PHEVs), and the intention of the research was to devise techniques for the reduction of overall cost: in a PHEV, power can either be taken from the battery or from combustion. Drawing power from the battery tends to age it, but drawing power from the IC combustion engine uses petrol-more expensive than mains electricity-and results in a higher running cost. The researchers were able both to characterise the trade-off and also to characterise the types of demand that led to the trade-off. The researchers' findings are relevant and provide some useful prerequisites for our work. By contrast, we will consider purely electric vehicles, and we will explicitly consider the trade-offs between cost and $\mathrm{CO}_{2}$ emissions.

\section{Scope of work}

In this paper, we will consider the impact of ageing on an EV powertrain sizing problem. We will be exploring two variables. Firstly, we will look to see how the sizing of a battery relates to its rate of degradation, and understand what the efficiency-aging trade-off looks like. Secondly, we will explore the use of supercapacitors to reduce degradation. We will explore the trade-offs between $\mathrm{CO}_{2}$ emissions and battery ageing, and also consider the economic cost. The results of this research will help manufacturers make informed decisions when designing EV battery systems. Our fundamental aim is to resolve some of the existing concerns relating to EV batteries, and thus help increase the uptake of ultra-low emission vehicles in the automotive industry worldwide. We will make some assumptions about ageing behaviour-our colleagues on the FUTURE Vehicles project will fill these gaps for us in due course-but the key point is the techniques we develop; these will be applicable to any battery model that represents degradation.

\section{THEORY}

In order to investigate our ageing phenomena, it is necessary for us to understand three things: how to model drive-cycles, how to model traction batteries, and how to model supercapacitors. We will discuss each in turn.

\section{Drive-cycle modelling}

In powertrain optimisation, we typically use simulations based on 'backwards modelling' (Figure 1) the models used are referred to either as 'backwards models' or 'backward-facing models'. These models use driving cycles (pre-determined velocity profiles) as their input, such as the New European Driving Cycle, and a typical model will work by allowing the user to scale different components of the system so as to get the best result (usually the lowest fuel consumption figure) [11].

Backward-facing models are a useful tool in vehicle optimisation, but there are several limitations [12]:

- To enable fast simulations, the time-stepping is relatively coarse, typically $1 \mathrm{sec}$;

- Because the velocity profile is fixed, backward-facing models cannot account for driver behaviour; 
- Many phenomena are approximated by 'quasi-static' maps, meaning that there is little or no representation of dynamic behaviour.

Despite their limitations, backward-facing models are a useful and widely-used. All models described in this paper will be backwards models.

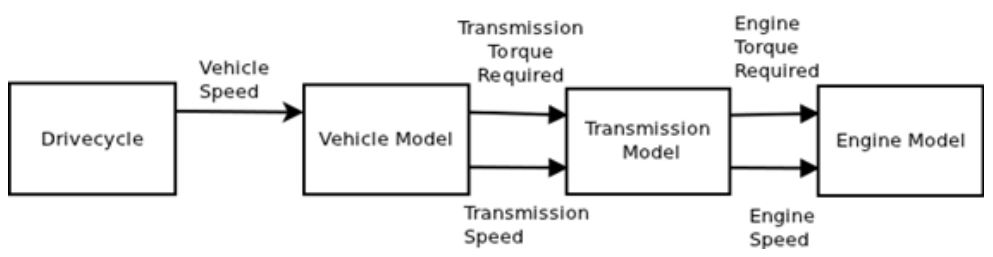

Figure 1. Backward-facing model

Diagram on Figure 1 shows the structure of a generic backwards-facing model. For our EV, we replace the 'engine' block with an electric machine connected to a battery in parallel with a supercapacitor.

\section{Battery modelling}

Battery models are traditionally based on simple equivalent circuits, often with parameters that vary based on the battery's state-of-charge. A typical structure used is the Thevenin model illustrated in Figure 2. (We have used this model structure, parameterised according to experimental data [13]. We have validated this against a 'known-good' model derived from another source [14] and confirmed that its behaviour is a close match.) The component values-the open source voltage $E_{0}$ and the resistances $R$, $R_{0}$-values are parameterised as polynomial functions of state-of-charge. (The capacitance $\mathrm{C}_{0}$ is constant.)
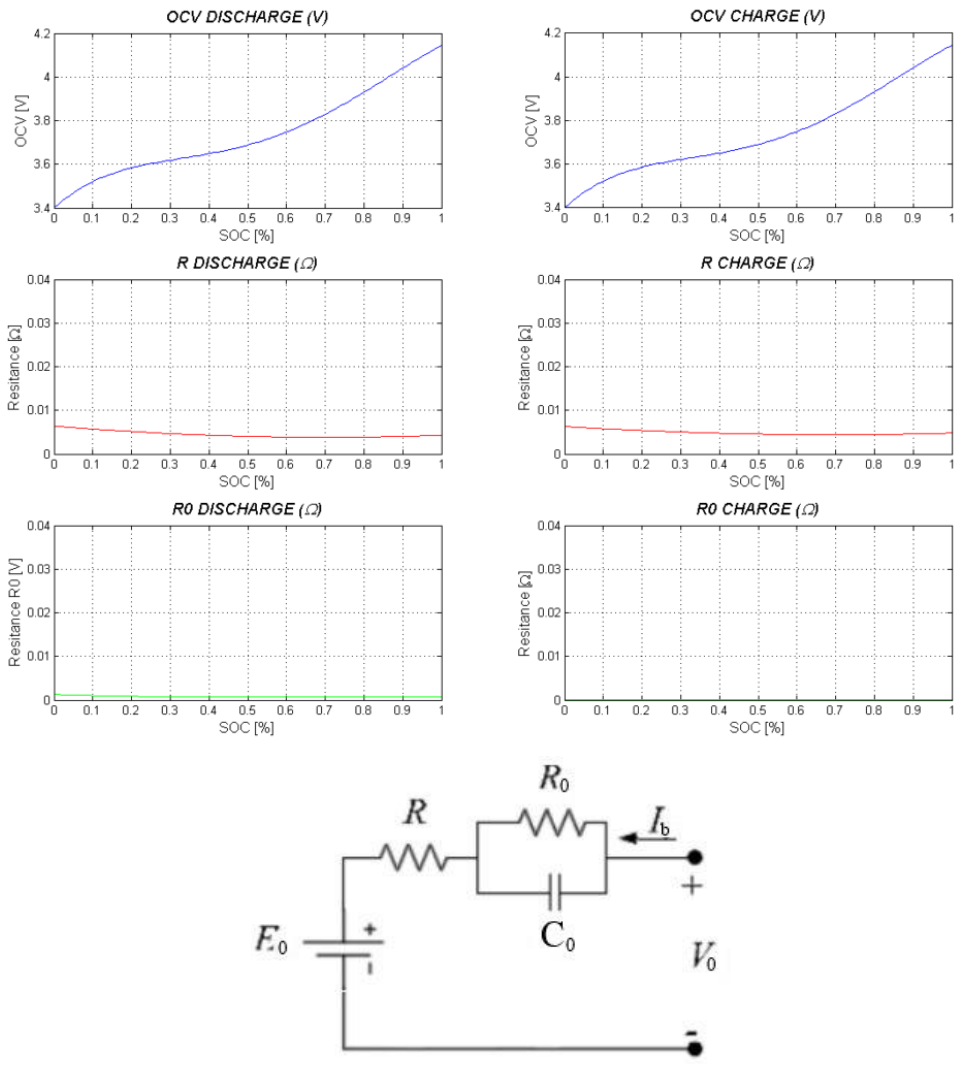

Figure 2. Equivalent battery circuit 
Notation on Figure 2 are given in text above. The component values are varied according to state-of-charge and current direction. When scaled for a single cell (as shown in all parameter graphs), the capacitance $\mathrm{C}_{0}$ has a constant value of $40 \times 10^{3} \mathrm{~F}$. Note that in practice the parameters will vary with age. We do not currently have access to characterisation data for aged batteries, so for simplicity we have not modelled parameter changes due to ageing.

The parameterization we have used in this model is exactly that defined in [13], and illustrated in Figure 2. The electrical circuit parameters vary as functions of temperature, current sense and state-of-charge. For simplicity, we have assumed that we are able to maintain a constant pack temperature of $25^{\circ} \mathrm{C}$ and that our model is scalable from a single cell to pack level. (We are in the process of developing a pack-level model that will incorporate realistic models of temperature effects, inter-cell resistances, etc., but we are dependent on the timing of a collaborative multi-partner research program for this. Our aim in this paper is to present the optimization techniques.)

Some sources overcomplicate the equivalent circuit analogy by introducing a second equivalent circuit to describe state-of-charge, but we feel this is unnecessary. Exactly the same behaviour can be implemented through a simple integral expression:

$$
\frac{d}{d t}(\mathrm{SOC})=\frac{+i_{\mathrm{b}}(t)}{3600 k_{\mathrm{c}}}
$$

where SOC is the state-of-charge, $i_{\mathrm{b}}$ is the current flowing into the battery and $k_{\mathrm{c}}$ is the battery capacity in [Ah]. Using a constant capacity this maintains simplicity, but the fact that the capacity is constant is not perfectly realistic: to improve on this we are in the process of developing a more sophisticated version of our model where $k_{\mathrm{c}}$ drops as the state-of-health worsens. (The factor of 3600 converts from [Ah] to equivalent base SI units; the choice of sign convention is arbitrary, and has been chosen to match the physical modelling convention where a positive current-voltage product corresponds to power absorption, as standard in our modelling environment: other sign conventions are equally good, though we felt it worth making ours clear in case it is counter-intuitive.)

Modelling state-of-health is more difficult, since there is less in the way of literature on the subject. US researchers have derived a nonlinear relationship for one of the main degradation modes (SEI layer growth) that we could use [10]; the same source also describes a control strategy for a hybrid vehicle that uses a simpler model, where ageing is simply proportional to the magnitude of the current flowing at the battery terminals. We have chosen to implement model similar to this:

$$
\frac{d}{d t}(\mathrm{SOH})=\frac{-\left|i_{\mathrm{b}}(t)\right|}{3600 k_{\mathrm{c}} \times 2 N_{\text {cycles }}}
$$

Here, $\mathrm{SOH}$ represents state-of-health (1 new, 0 'bad'). In practice, a 'bad' battery is one whose capacity has dropped to $80 \%$ of its original value. (For this work package, we have only modelled the age of the battery, not its effect on the parameter values, but it is easy to extend our models to account for this; we have indeed done so for some estimation work.) The important thing our model tells us is how much a given current profile will degrade the battery. $k_{\mathrm{c}}$ is the battery capacity (as before) and $N_{\text {cycles }}$ is the number of charge-discharge cycles a new battery can take before it reaches zero state-of-health.

It should be clear to the reader that the ageing model in this battery is assumed and it is unlikely to account for every possible degradation mechanism. However, it is a useful starting point and we can use it to demonstrate the techniques we will use as our models improve. 
So far we have dealt with the behaviour of the batteries, but we are also interested in the cost. There are several ways to account for this, but we have chosen to consider the cost to the end-consumer. This may be estimated from the expression:

$$
\text { battery cost }(\$)=(22.0 / \mathrm{kW}) P_{\mathrm{B}(\max )}+(700.0 / \mathrm{kWh}) W_{\mathrm{B}}+680.0
$$

where $P_{\mathrm{B}}$ is the battery pack rated power and $W_{\mathrm{B}}$ is the pack rated capacity [15].

\section{Supercapacitor modelling}

The idea of using supercapacitors in vehicles is not new. Sometimes they are considered as an alternative to batteries, to be used in conjunction with another power source such as a fuel cell [16]. The literature contains at least one (non-EV specific) example of a 'hybrid' power pack comprising supercapacitors as well as conventional batteries, providing a better-than usual compromise between conflicting energy density and power density requirements [17]. In an electric vehicle context. Super capacitors have been successfully deployed to boost the power capability of a ZEBRA battery pack [18]. The impact of the topology of the power electronics and their governing power electronics has been studied [19]. It is generally accepted in the literature that short-term high currents are damaging to batteries and that supercapacitors can be used to remove damaging stress on batteries. To date, the authors are not aware of any studies that explicitly model the degree of ageing in a hybrid vehicle energy storage pack, where supercapacitors are used to offset the damage. In this paper we intend to demonstrate such a technique. Although the exact results are limited by our simplified battery model, the general techniques we develop will be applicable to more realistic models, and there is thus a potential for useful results.

Super capacitors are essentially large, high-power capacitors. There is plenty of material freely available describing their construction and properties. In many ways, their behaviour is identical to that of a conventional capacitor. However, because they are larger, there is typically a difference in charge distribution near the device surface and deep within. Since the behaviour of super capacitors is not the present focus of our work, we have chosen to model them simply as a capacitor in series with a resistor. (This is often the best one can do just based on a manufacturer's data sheet!) We intend to refine this model over time, though, since the purpose of our exercise is to demonstrate the general technique, our results are in any case tentative; we feel that the most likely improvement that we would need to make to our model would be the introduction of a series inductance. We have also neglected 'pack-level' effects in our super capacitor pack (e.g. power electronics required for balancing and energy management, leakage, etc.) Since the super capacitor is likely to be used only for short, transient energy bursts, leakage may not be significant, but the efficiency of the cell management system has not been explored. These are limitations in the model, but-as for the battery-it is not the absolute result, but rather the technique that we are trying to illustrate in this paper.

We have described the key components of our simulation. There are of course other components that we have not described such as the vehicle dynamics and the electric machine characteristics. These are 'industry standard' and follow the pattern one might find in any good text book of the subject.

We have assumed a supercapacitor array constructed of Maxwell's PC2500, a commercially available device with published characteristics [20]. The cost of this device is approximately $\$ 30$, and this can scaled up according to the array size. Also, we will assume that supercapacitors do not age significantly. (From conversations with researchers in the field, we believe that this is a reasonable assumption though one should 
note that Maxwell specify a useful life of 10 years in their data sheet.) The PC2500 has a capacitance of $2700 \mathrm{~F}$, a rated voltage of $2.5 \mathrm{~V}$ (continuous) $/ 2.7 \mathrm{~V}$ (peak), a series resistance of $1.0 \mathrm{~m} \Omega$ at DC, a rated current of $625 \mathrm{~A}$ and a leakage current of $6 \mathrm{~mA}$. Further data is available on the manufacturer's data sheet [20].

\section{VARYING BATTERY SIZE}

\section{Method}

For this part of the work, we considered a model based on the Smart ed. (There was no particular reason for this other than it being readily available to us.) We varied the number of cells in our battery pack (both the number of 'strings' in parallel, and the length of the strings). For each configuration, we ran the model in charge-discharge cycles for $20,000 \mathrm{~km}$ (long enough to see some degradation, but short enough to simulate reasonably quickly) measured the battery degradation, and used this to estimate the useful range of the battery. We also estimated the $\mathrm{CO}_{2}$ emissions assuming a relatively clean electricity supply: the exact scaling chosen is arbitrary, designed to give figures that 'feel' comparable with the conventional gasoline figures we are used to working with. If we were comparing our results with a gasoline vehicle, we would need to revisit this part of the work taking careful account of the true well-to-tank emissions figure. (This will of course vary in different parts of the world.) Since we are comparing only electric vehicles with electric vehicles, and the scaling factor is common to all, a significant error can be tolerated here: it is merely present to help meaningful comparisons within this paper.

The model was driven with a 'combined' driving cycle made up of the urban, road, motorway, road and urban sections of the well-known Artemis cycle [21].

\section{Results}

The results are illustrated in Figure 3. The plots show that $\mathrm{CO}_{2}$ emissions are very poor for small batteries. As battery size increases, $\mathrm{CO}_{2}$ emissions initially fall, then begin to increase again as battery size increases. Lifespan tends to increase as battery size increases. The effective battery price (normalised with respect to the lifetime) is poor for small batteries, then relatively flat.

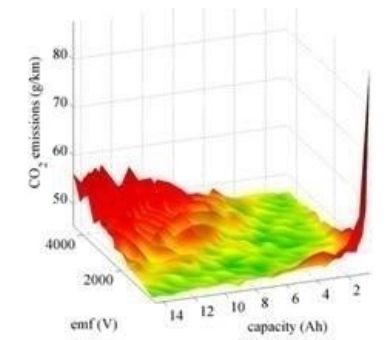

(a) $\mathrm{CO}_{2}$ emissions vs. battery size

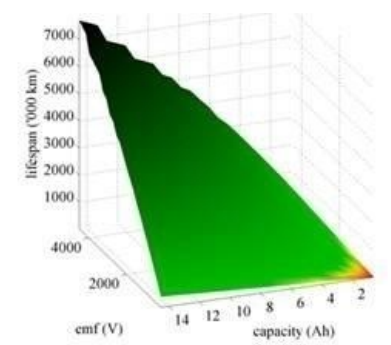

(b) Lifespan vs. battery size

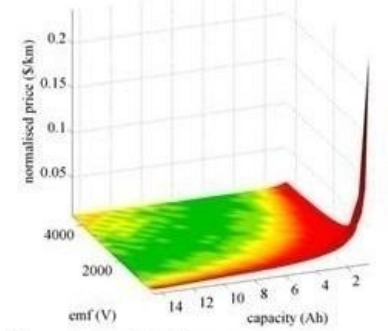

(c) Normalised cost vs. battery size

Figure 3. Variation of vehicle performance measures with battery size 
Plots in Figure 3 show how the $\mathrm{CO}_{2}$ emissions, lifespan, and powertrain cost per unit lifespan vary as functions of the battery size. (Note that the largest batteries are deliberately oversized and have unusually high voltages. As described in the text, this is simply to show a trend, not for a practical implementation.)

The reader should note that we have deliberately emphasised the 'voltage' beyond what would be considered a 'normal' region: EVs typically operate with voltages under $600 \mathrm{~V}$, and we have only 'pushed' the voltages higher than this in order to see the shape of the trend that emerges. There would be practical problems implementing such a high-voltage system, not in the least the non-standard nature of the hardware required.

\section{Discussion}

Any results that we produce are heavily dependent on the assumptions we have made in our model. Although our ageing model is sensible given the data available to us, we need to be mindful that it is an approximation of what we know, and what we know is incomplete. We expect to revisit our results in due course. These caveats should be kept in mind: it would be unwise to attempt radical changes to battery production without waiting for confirmation that the patterns observed in this paper apply in reality.

From the variation of the battery size, we see that for our model of ageing, batteries will last longer if they are oversized. The smallest battery-which will be the cheapest-is not good value when taken over the lifetime of the battery. The smallest battery is also poor for emissions. There seems to be a region where it is possible to simultaneously achieve near optimal $\mathrm{CO}_{2}$ emissions and good value.

It is obvious that large batteries will require more $\mathrm{CO}_{2}$ than small ones. At first glance, it might not be obvious why small batteries achieve poor $\mathrm{CO}_{2}$ performance. There is, however, a good reason: if a battery is delivering close to its maximum rated power, a greater element of the stored energy will be dissipated in the battery itself (due to higher currents passing through the internal resistances). It is worth noting that that we had to use very large batteries-probably much larger than we could actually put on a vehicle-to see the battery mass having a significant detrimental effect.

It is also notable that the surfaces we have produced are quite uneven. It is possible that this is an artefact of the discontinuous nature of drive cycles and charging patterns: some batteries can tolerate more drive cycles before needing a recharge than others: this needs further investigation. The presence of many local minima and maxima could make automatic optimisation challenging.

In a sensible price range, it is likely that the most dominant trade-off will be between price and lifespan. For acceptably-priced batteries with a reasonable lifespan, the $\mathrm{CO}_{2}$ emissions appear reasonably constant (Figure 4).

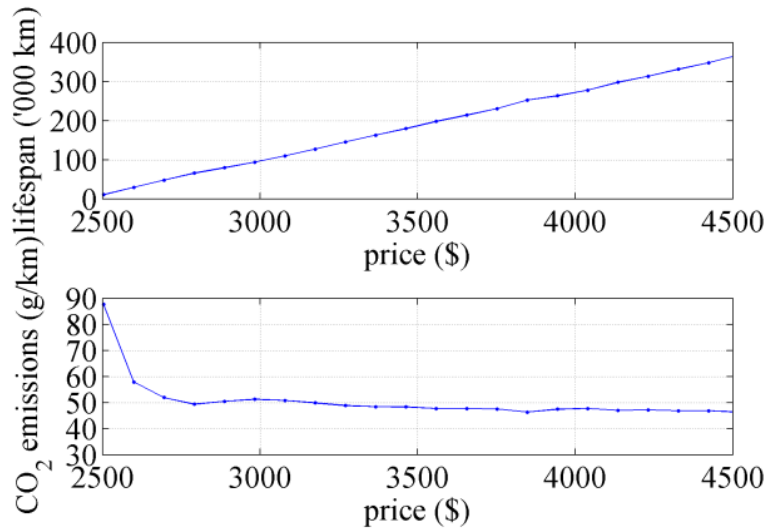

Figure 4. Battery trade-offs 
Over a sensible price range, battery lifespan increases with price but $\mathrm{CO}_{2}$ remains flat. The most dominant trade-off is between lifespan and cost.

\section{ADDING SUPERCAPACITORS}

\section{Method}

This part of the work used a similar model (this time parameterised to match the Nissan Leaf). This time, instead of varying the size of the battery, we added a supercapacitor pack and varied its size as for the battery pack. We recorded the same data: overall range, $\mathrm{CO}_{2}$ emissions and vehicle cost.

The algorithm used was not optimised: essentially, during regenerative braking, if there was room in the supercapacitor, we stored energy in it in preference to the battery; during driving phases, if there was energy in the supercapacitor, we used it in preference to the battery. (In extreme braking situations, we used friction brakes alone.) This algorithm is a sensible starting point as, though it is likely that one could do better: this will be an area of future research.

For this section of work, the model was driven with the industry-standard New European Driving Cycle (NEDC).

\section{Results}

The results are illustrated in Figure 5. The plots show that there is a near-optimal convex 'valley' in the $\mathrm{CO}_{2}$ emissions, corresponding to a concave 'ridge' in lifespan and another convex valley in the normalised (cost per unit distance).

For comparison, the performance of an equivalent vehicle with no supercapacitor pack was evaluated: for this, the $\mathrm{CO}_{2}$ emissions were $77.32 \mathrm{~g} / \mathrm{km}$, the battery lifespan was $156,120 \mathrm{~km}$, and the powertrain cost per kilometre was $\$ 0.090$.

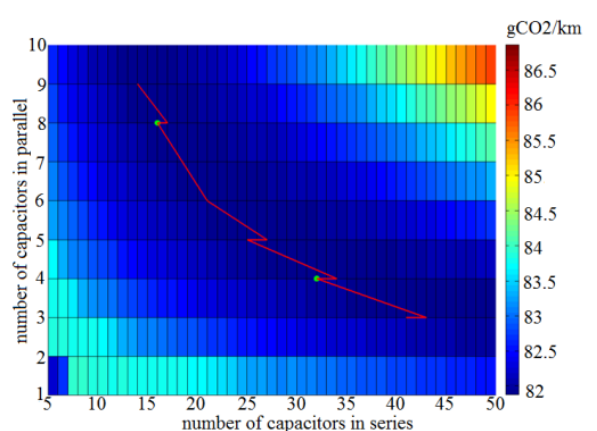

(a) $\mathrm{CO}_{2}$ emissions vs. array size

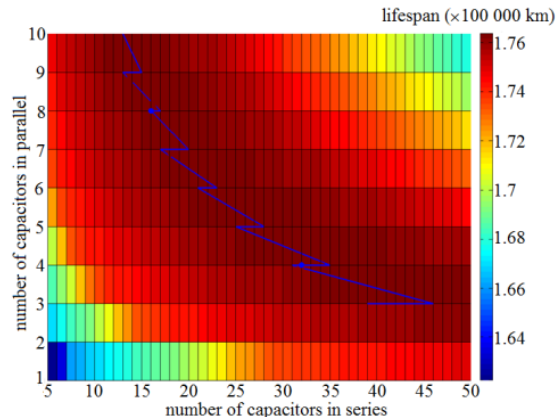

(b) Lifespan vs. array size

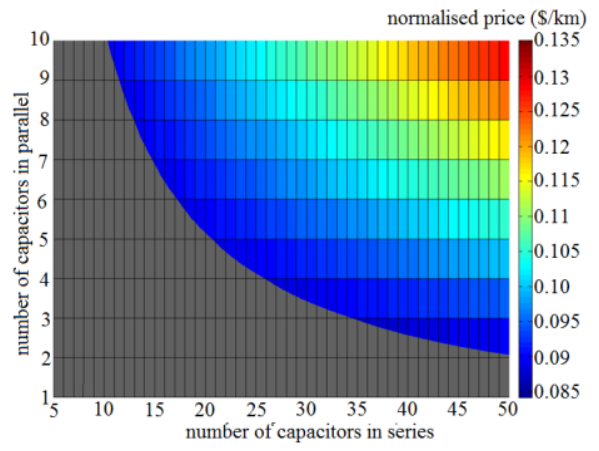

(c) Normalised cost vs. array size

Figure 5. Variation of vehicle performance measures with super capacitor array size 
These plots show how the $\mathrm{CO}_{2}$ emissions, lifespan, and cost per unit lifespan vary as functions of the supercapacitor array size. (In the bottom plot, solutions that might reduce the overall vehicle cost are highlighted in grey.) These plots suggest that good $\mathrm{CO}_{2}$ emissions and good battery lifespan go together.

\section{Discussion}

It is difficult to present three-dimensional graphs well, particularly in the space of a conference paper. Nevertheless, there are some interesting features of the results. It is notable that (as for the battery size variation) there is no reason to think that requirements for low emissions, good battery durability and good value are in conflict. As a test case, we considered a vehicle with a $4 \times 23$ super capacitor array. This would give a vehicle with the following characteristics:

- $\mathrm{CO}_{2}$ emissions $82.0 \mathrm{~g} / \mathrm{km}$;

- Battery lifespan 176,040 km;

- Powertrain cost per km $\$ 0.089$ ('amortised' over battery lifespan).

In essence, without decreasing the 'value' of the powertrain, the lifespan has been increased by $20,000 \mathrm{~km}$. This represents a significant improvement in battery durability. It would be necessary to convince consumers that paying more 'up front' would be in their interests: this would depend somewhat how expectations of vehicle price and expected mileage trade off against each other. There is a small additional $\mathrm{CO}_{2}$ cost, but this may well be regarded as tolerable: if in future the vehicle was powered by $100 \%$ renewable sources, the additional $\mathrm{CO}_{2}$ cost would become zero.

As for the battery case, the results are heavily dependent on the modelling assumptions, and further work is needed to see if they apply in the real-word. The techniques we have demonstrated will be useful going forward.

At present, we have not attempted to optimise the supercapacitor utilisation algorithm. This may result in additional gains, and it would be possible to extend this to the effects of regenerative braking. Also, we have not combined the optimisation of battery sizing, array size and other vehicle aspects together. This would be an interesting study, and we will explore it in our future work.

\section{CONCLUSIONS}

In this paper, we have achieved the following:

- We have described key elements of a 'backward facing' model that allows us to evaluate various properties of an electric vehicle powered by a combination of a battery and supercapacitors. This model allows us to consider $\mathrm{CO}_{2}$ emissions, battery ageing and power train cost;

- We have presented a simple nonlinear model of battery ageing, using representations of battery behaviour from the literature and an assumed ageing model. We plan to replace this with a model with a stronger basis in fact which we will receive from our colleagues in due course, but for the time being it is a useful way to test optimisation and analysis techniques;

- We have used our models to perform analyses of the effects of varying the size of a battery and (separately) a supercapacitor array, and shown how these may be used to understand the relationships between $\mathrm{CO}_{2}$ emissions, battery ageing and power train cost;

- For our assumed model, we have found that there is no strong conflict between the properties we want to optimise: in particular, for the supercapacitor array, good $\mathrm{CO}_{2}$ emissions, good battery lifespan and low powertrain cost (amortised over 
vehicle lifespan) all come together. However the absolute powertrain cost may be a constraint;

- We have noted that because of our modelling assumptions, the above results are not definitive, and we will need to do further work to see if the results hold in reality. (The techniques we developed to get the results are, however, useful and reusable.)

Our future plans include bringing the sizing of the battery pack and the supercapacitor together into a single optimisation, incorporating emerging models describing degradation, and applying optimisation to the algorithms that decide when to use the battery, the supercapacitor and friction brakes. We will also conduct sensitivity analyses to see how dependent the results are on the assumptions we have made and to understand their real-world risks and benefits.

\section{ACKNOWLEDGEMENT}

The authors wish to thank EPSRC, who supported this work as part of the FUTURE Vehicles project.

\section{REFERENCES}

1. Royal Society, Climate Change: A Summary of the Science, September 2010.

2. NASA, Climate Change: How do we know ?, http://climate.nasa.gov/evidence, [Accessed: 8-August-2013]

3. European Parliament, European Parliament resolution of 24 October 2007 on the Community Strategy to reduce $\mathrm{CO}_{2}$ Emissions from Passenger Cars and Light-commercial Vehicles (2007/2119(INI)), Official Journal of the European Union, C263 E/433, Brussells: European Parliament, 2008.

4. Department of Energy and Climate Change, The Carbon Plan: Delivering our low Carbon Future, London: Department of Energy and Climate Change, 2011.

5. Vaughan, A., Electric Car sales accelerated in 2012, The Guardian, 8 January 2008.

6. Mitsubishi UK web site, http://www.mitsubishi-cars.co.uk, [Accessed: 7-August-2013]

7. Nissan UK web site, http://www.nissan.co.uk, [Accessed: 7-August-2013]

8. FUTURE Vehicles web site, http://www.futurevehicles.ac.uk, [Accessed: 7-August-2013]

9. Troxler, Y., Wu, B., Marinescu, M., et al., The effect of Thermal Gradients on the performance of Lithium-ion Batteries, Journal of Power Sources, Vol. 247, pp 1018-1025, 2013, http://dx.doi.org/10.1016/j.jpowsour.2013.06.084

10. Moura, S. J., Stein, J. L. and Fathy, H. K., Battery-Health Conscious Power Management in Plug-in Hybrid Electric Vehicles via Electrochemical Modelling and Stochastic Control, IEEE Transactions on Control System Technology, Vol. 21, No. 3, pp 679-694, May 2013, http://dx.doi.org/10.1109/TCST.2012.2189773

11. G. Rizzoni, G., Guzzella, L. and Baumann, B.M., Unified modelling of Hybrid Electric Vehicle Drivetrains, IEEE/ASME Transactions on Mechatronics, Vol. 4, pp 246-257, 1999, http://dx.doi.org/10.1109/3516.789683

12. Wipke, K. B., Cuddy, M. R. and Burch, S. D., ADVISOR 2.1: A User-friendly Advanced Powertrain Simulation using a Combined Backward/forward approach, IEEE Transactions on Vehicular Technology, Vol. 48, pp 1751-1761, 1999, http://dx.doi.org/10.1109/25.806767

13. Antaloae, C., Marco, J. and Assadian, F., A Novel Method for the Parameterisation of a Li-Ion Cell Model for EV/HEV Control Applications, IEEE Transactions on Ve- 
hicular Technology, Vol. 61, No. 9, pp 3881-3892, November 2012, http://dx.doi.org/10.1109/TVT.2012.2212474

14.Tremlay, O., Essaint, L. A. and Dekkiche, A. I., A generic Battery Model for the Dynamic Simulation of Hybrid Electric Vehicles, Proceedings of the IEEE Vehicle Power and Propulsion Conference, Arlington, Texas, 9-12 September 2007.

15.O Keefe, M., Brooker, A., Johnson, C., et al., Battery Ownership Model: A Tool for Evaluating the Economics of Electrified Vehicles and Related Infrastructure 2010, Proceedings of the 25th International Battery, Hybrid and Fuel Cell Electric Vehicle Symposium and Exposition, Shenzhen, China, 5-9 November 2010.

16.Thounthong, P. and Rael, S., The Benefits of Hybridization, IEEE Industrial Electronics Magazine, Vol. 3, No. 3, pp 25-37, 2009, http://dx.doi.org/10.1109/MIE.2009.933885

17.Kupperman, A. and Aharon, I., Battery-Ultracapacitor Hybrids for Pulsed Current Loads: A Review, Renewable and Sustainable Energy Reviews, Vol. 15, No. 2, pp 981-992, 2011, http://dx.doi.org/10.1016/j.rser.2010.11.010

18.Dixon, J., Nakashima, I., Arcos, E. F. and Ortúzar, M., Electric Vehicle using a Combination of Ultracapacitors and ZEBRA Battery, IEEE Transactions on Industrial Electronics, Vol. 57, No. 3, pp 943-949, 2010, http://dx.doi.org/10.1109/TIE.2009.2027920

19.Amjadi, Z. and Williamson, S. S., Power-electronics-based Solutions for Plug-in Hybrid Electric Vehicle Energy Storage and Management Systems, IEEE Transactions on Industrial Electronics, Vol. 57, No. 2, pp 608-616, 2010, http://dx.doi.org/10.1109/TIE.2009.2032195

20. Maxwell Technologies, PC2500 BOOSTCAP Ultracapacitor Data Sheet, Undated [Accessed: 2013]

21.André, M., The ARTEMIS European driving Cycles for measuring Car Pollutant Emissions, Science of the Total Environment, Vol. 73-85, pp 334-335, 2004, http://dx.doi.org/10.1016/j.scitotenv.2004.04.070 